\title{
A Consolidated Method for Selective Isolation of Actinomycetes Based on Choice of Substrate
}

\author{
Deepika Tiwari ${ }^{1}$, Shobha Shouche ${ }^{1}$, Praveesh Bhati $^{2 *}$, Preeti Das ${ }^{3}$ \\ ${ }^{1}$ Govt. Madhav Science P.G. College, Ujjain (M.P.), India \\ ${ }^{2}$ State Forensic Science Laboratory, Sagar, (M.P.), India \\ ${ }^{3} \mathrm{SOS}$ in Microbiology, Vikram University, Ujjain (M.P.), India \\ *Corresponding author email: bhati_p212@yahoo.co.in \\ Received: 13 March 2021 / Revised: 20 November 2021 / Accepted: 07 December 2021 / Published: 11 December 2021
}

\begin{abstract}
Actinomycetes are known as filamentous, Gram positive bacteria. They form the majority of the microbial load in various niches; soils, composts, etc. The study aimed to evaluate the method of isolating slow growing actinomycetes from four different sources: garden soil, cow dung manure compost, floral waste compost, and floral waste vermicompost. In this study, an integrated method consisting of physical and chemical pretreatment of the sample and the use of selective media was used to isolate actinomycetes. Physical treatment includes air drying, sun drying, dry heating in an oven, and moist heat treatment, whereas chemical treatment includes enrichment of the sample with $\mathrm{CaCO}_{3}$ followed by plating on actinomycetes-specific media with the incorporation of antibacterial and antifungal antibiotics. The actinomycetes count on the plate was reported in CFU/gm of dry wt. Morphological and microscopic characteristics of purified isolates were noted. The results were compared, and it was found that the pretreatment method of a particular sample depends on the choice of substrate. Overall, physical treatment followed by chemical enrichment showed relatively higher counts on the plate and better results. Here we also found the dominance of fluorescent Pseudomonas sp. in case samples from vermicompost. The study can be of great importance in isolating novel and rare genera of actinomycetes. These methods can help speed up the isolation and screening of novel actinomycetes which will ultimately be important for the discovery of antibiotics and other industrially vital bioactive compounds.
\end{abstract}

Keywords: Actinomycetes, Pre-treatment, Enrichment.

\section{Introduction}

Actinomycetes are aerobic gram-positive bacteria, filamentous in nature with a fungal type of morphology. They are one of the major groups of soil population, widely distributed in nature and inhabit various niches other than terrestrial soil such as marine sediments, mangrove environment, composts, vermicomposts etc (Yami et al., 2003; Baniya and Vaidya, 2011; Mohseni et al., 2013; Gopalkrishnan et al., 2014; Mohanta, 2014). They are saprophytic in nature and play an important role in decomposition of waste and recycling of organic matter (Goodfellow and Williams, 1983). They have DNA rich in GC content, 57-75\% (Lo et al., 2002). They have the ability to produce antibiotics of agriculture and medicinal use. About $80 \%$ of the world's antibiotics are known to come from actinomycetes, mostly from the genera Streptomyces and Micromonospora (Pandey et al., 2004). They have also been recognized as prolific producers of enzymes such as amylase, lipase, protease (Lekshmi et al., 2014), cellulase (Mohanta, 2014), glucose isomerase (Sheetal and Modi, 2013) etc. of commercial interest, plant hormones (Gopalkrishnan et al., 2014) and other secondary metabolites. Thus, they have allured considerable research interest. They are the part of microbial flora of most natural substrates (El-Nakeeb and Lechevalier, 1962). Numerous methods have been suggested to facilitate the isolation of actinomycetes and to separate them from other non-actinomycetes bacteria and fungi. Isolation of actinomycetes itself is a challenging task (Williams and Cross, 1971). They need to be cultivated than to be cultured. These methods include pretreatment of samples and use of selective media 
Tiwari et al., Int. Ann. Sci.; Vol. 11, Issue 1, pp: 10-21, 2021

for increasing the slow growing actinomycetes flora from substrate before plating out. Pretreatment of sample can be physical such as air drying at room temperature (Salim et al., 2017), sun drying (Sheetal and Modi, 2013), dry heat in oven (Abidin et al., 2016), moist heat treatment (Mohseni et al., 2013), centrifugation etc (El-Nakeeb and Lechevalier, 1962) or can be chemical such enriching the sample with 1\% CaCO3 (Tsao et al., 1960) or chitin etc. Lechevailer (1963) suggested that $\mathrm{CaCO} 3$ treatment gave highest colony counts. Physical treatment together with chemical treatment favors growth of actinomycetes and reduction in the number of other microbes. Selective media supplemented with antibiotics have been used by many researchers to separate actinomycetes from other bacteria and fungi. These antibiotics do not affect the growth of actinomycetes and thus can be successfully used (El-Nakeeb and Lechevalier, 1962). The purpose of our present study is to compare various pretreatment methods and find out the method that can give relatively higher counts of actinobacteria on plate.

\section{Materials and Methods}

\subsection{Sample Collection}

Soil samples were collected from garden nearby village school campus. For isolation of actinomycetes soil was to be taken 6-12 inches at depth (Iyer et al., 2014). Four different samples were taken in this study 1) Soil samples 2) 20 days old cow dung left to degrade, turns black 3) Sample from compost prepared from floral waste and cow dung in ratio 1:1 (2kg each) matured in 60 days (approx.). Sample from vermicompost prepared from floral waste and cow dung in ratio 1:1 kg each (Shouche et al., 2011) matured in 46-50 days (approx.). $400 \mathrm{gm}$ of mature compost and vermicompost sample kept for further processing was used in this study. All the samples were collected by maintaining aseptic conditions.

\subsection{Pretreatment of Sample}

All the four samples were pretreated before plating out on selective media (Table 1). Samples without pretreatment were kept as control. The selective isolation was done using following combinations mentioned in table below.

Table 1: Selective isolation of actinomycetes

\begin{tabular}{|c|c|c|}
\hline Pretreatment of sample & Selective media & $\begin{array}{c}\text { Antibacterial and antifungal } \\
\text { supplements }\end{array}$ \\
\hline No treatment (control) & SCA & - \\
\hline No treatment (control) & AIA & - \\
\hline No treatment (control) & SCA & CHL and ICZ \\
\hline No treatment (control) & AIA & CHL and ICZ \\
\hline \multicolumn{3}{|l|}{ Dry treatment } \\
\hline Air drying at RT & SCA & - \\
\hline Air drying at RT & AIA & - \\
\hline Air drying at RT & SCA & CHL and ICZ \\
\hline Air drying at RT & AIA & CHL and ICZ \\
\hline Sun drying & SCA & - \\
\hline Sun drying & AIA & - \\
\hline Sun drying & SCA & CHL and ICZ \\
\hline Sun drying & AIA & CHL and ICZ \\
\hline \multicolumn{3}{|l|}{ Wet treatment } \\
\hline Air drying at RT + heating in water bath at $60^{\circ} \mathrm{C}$ & SCA & CHL and ICZ \\
\hline Air drying at RT + heating in water bath at $60^{\circ} \mathrm{C}$ & AIA & CHL and ICZ \\
\hline Sun drying + heating in water bath at $60^{\circ} \mathrm{C}$ & SCA & CHL and ICZ \\
\hline Sun drying + heating in water bath at $60^{\circ} \mathrm{C}$ & AIA & CHL and ICZ \\
\hline \multicolumn{3}{|l|}{ Chemical enrichment } \\
\hline Sample enriched with $\mathrm{CaCO}_{3}$ for 7 days and air dried & SCA & CHL and ICZ \\
\hline Sample enriched with $\mathrm{CaCO}_{3}$ for 7 days and air dried & AIA & CHL and ICZ \\
\hline
\end{tabular}

Note: RT= Room Temperature, SCA = Starch Casein Agar, AIA = Actinomycete Isolation Agar, CHL = Chloramphenicol, ICZ = Itraconazole. 
The Starch Casein Agar (SCA) and Actinomycete Isolation Agar (AIA) used here were procured from HiMedia, Mumbai, India. For chemical enrichment, the particular sample was mixed with for $1 \%$ of $\mathrm{CaCO}_{3}$ (Tsao et al., 1960). It was kept on filter paper moistened with sterile water in a petridish for 7 days and then air dried for further use. All the pretreated samples were crushed into powder and sieved and were subjected to serial dilution up to $10^{-6}$ before plating on agar medium.

\subsection{Isolation of Actinomycetes from Pretreated Samples}

For the isolation of actinomycetes $10 \mathrm{gm}$ of dried pretreated sample was added to $90 \mathrm{ml}$ of sterile water in conical flask and shaken in vortex mixer vigorously for detachment of spore chains (Workie and Abate, 2016). It is then allowed to settle for 10 minutes. From the suspension $1 \mathrm{ml}$ was taken and added to $9 \mathrm{ml}$ of sterile water followed by serial dilution up to $10^{-6}$ of which $0.1 \mathrm{ml}$ was spread using sterile $\mathrm{L}$ - shaped glass rod on SCA (Kuster and Williams, 1964) and AIA (Abidin et al, 2016) supplemented with or without antibiotics. The antibiotics used in the medium were Chloramphenicol - $50 \mu \mathrm{g} / \mathrm{ml}$ as an antibacterial drug (Williams and Davies, 1965) and Itraconazole $-75 \mu \mathrm{g} / \mathrm{ml}$ as an antifungal drug (Peela and Porana, 2017). After plating the sample plates were kept inverted at $30^{\circ} \mathrm{C}$ for 7-14 days (Kuster and Williams, 1964; Jeffrey, 2008; Anusuya and Geetha, 2012). In case of moist heat pretreatment, the process is same as mentioned above except serially diluted soil suspensions before plating were kept in water bath for $50^{\circ} \mathrm{C}$ for $10 \mathrm{~min}$ (Arifuzzaman et al., 2010; Mohseni et al., 2013) Colonies with tough or powdery texture, dry or folded appearance and branching filaments, substrate or aerial mycelium were sub cultured on SCA for further studies.

\subsection{Microscopic Characterization}

Microscopy was performed with simple stain technique using methylene blue was used (Kahasabuli and Kibera, 2014). A mass of pure colony of isolate was picked or scooped using a sterile wire loop, spread on clean glass slide, air dried and fixed by passing over flame gently. The slide was the flooded with methylene blue stain and after few seconds excess stain washed off in running tap water. The slide was dried using blotting paper and observed using a light microscope under 10X, 40X and also under 100X using immersion oil. Nature of hyphae and spore chain was observed and identified according to Shirling and Gottlieb, 1966 and photograph was taken.

\section{Results}

From the four different samples collected actinomycetes isolated were represented as CFU/g dry wt. (Table $2,3,4,5)$. Soil Samples without treatment showed no growth of actinomycetes in any of the media SCA or AIA (Table 2, Fig. 1).

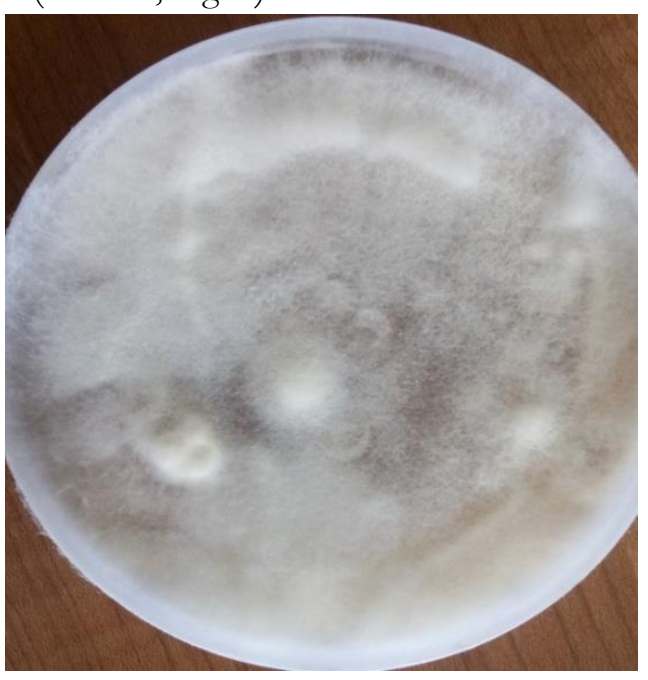

Figure 1: Plate with sample pretreatment and without $A b-$ no actinomvcetes visible

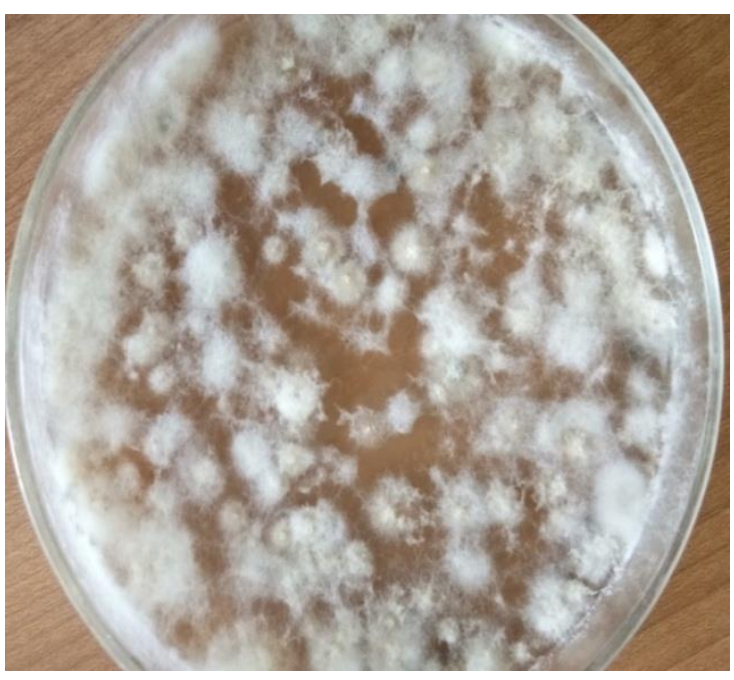

Figure 2: Plate with sample pretreatment and without Ab 
Tiwari et al., Int. Ann. Sci.; Vol. 11, Issue 1, pp: 10-21, 2021

Samples without treatment but with use of antibiotics also showed no growth of actinomycetes (Table 2 Fig. 2). Similar is the case with other cowdung compost, floral compost and floral vermicompost. Samples with physical treatment such as air dry and sun drying along with the use of antibiotics showed some actinomycetes isolates and fungal inhibition (Fig 3). Results showed that highest no. of actinomycetes count was achieved when subjected to both dry and wet heat treatment followed by plating on SCA and AIA agar supplemented with antibiotics (Fig 1-7). However, in case of cow dung compost actinomycetes count was better with air dried sample at room temperature on agar medium with antibiotics. The bacterial counts were higher when the sample was subjected to $\mathrm{CaCO}_{3}$ enrichment (Fig $4 \& 5$ ). Hence chemical enrichment showed better results in this case. On the other hand, in case of floral waste compost good results were obtained only when sample enriched with $\mathrm{CaCO}_{3}$ (Table 3).

Table 2: Selective isolation of actinomycetes from soil near plant area:

\begin{tabular}{|c|c|c|c|c|}
\hline $\begin{array}{l}\text { Pretreatment } \\
\text { of sample }\end{array}$ & Selective Media & $\begin{array}{l}\text { Growth of } \\
\text { actinomycetes }\end{array}$ & Growth of other microbes & $\begin{array}{l}\text { CFU/gm } \\
\text { of dried } \\
\text { sample }\end{array}$ \\
\hline No treatment & SCA & No growth & Bacterial and fungal contamination & - \\
\hline No treatment & AIA & No growth & Bacterial and fungal contamination & - \\
\hline No treatment & $\mathrm{SCA}+\mathrm{CHL}+\mathrm{ICZ}$ & $\begin{array}{l}\text { No distinct } \\
\text { actinomycetes } \\
\text { appear }\end{array}$ & $\begin{array}{l}\text { No bacteria; fungal contamination of } \\
\text { Aspergillus niger }\end{array}$ & - \\
\hline No treatment & $\mathrm{AIA}+\mathrm{CHL}+\mathrm{ICZ}$ & $\begin{array}{l}\text { No distinct } \\
\text { actinomycetes }\end{array}$ & $\begin{array}{l}\text { No bacteria; } \\
\text { fungal contamination of Aspergillus } \\
\text { niger, Aspergillus fumingatus, green } \\
\text { mold }\end{array}$ & - \\
\hline \multicolumn{5}{|l|}{ Dry treatment } \\
\hline Air dried at RT & SCA & No growth & Bacterial and fungal contamination & - \\
\hline Air dried at RT & AIA & No growth & Bacterial and fungal contamination & - \\
\hline Air dried at RT & SCA + CHL+ICZ & Poor growth & Aspergillus niger contamination & $2 \times 10^{-5}$ \\
\hline Air dried at RT & $\mathrm{AIA}+\mathrm{CHL}+\mathrm{ICZ}$ & Poor growth & $\begin{array}{l}\text { Aspergillus niger contamination; } \\
\text { But other fungi is inhibited }\end{array}$ & $1 \times 10^{-5}$ \\
\hline Sundried & SCA & No growth & Bacterial and fungal contamination & - \\
\hline Sundried & AIA & No growth & Bacterial and fungal contamination & - \\
\hline Sundried & $\mathrm{SCA}+\mathrm{CHL}+\mathrm{ICZ}$ & Poor growth & $\begin{array}{l}\text { Aspergillus niger contamination; but } \\
\text { other fungi is inhibited }\end{array}$ & $2 \times 10^{-5}$ \\
\hline Sundried & $\mathrm{AIA}+\mathrm{CHL}+\mathrm{ICZ}$ & Poor growth & $\begin{array}{l}\text { Aspergillus niger contamination; } \\
\text { but other fungi is inhibited }\end{array}$ & $2 \times 10^{-5}$ \\
\hline \multicolumn{5}{|l|}{$\begin{array}{l}\text { Wet } \\
\text { treatment }\end{array}$} \\
\hline $\begin{array}{l}\text { Air dried at RT } \\
+ \text { heated in } \\
\text { water bath for } \\
60{ }^{\circ} \mathrm{C}\end{array}$ & $\mathrm{SCA}+\mathrm{CHL}+\mathrm{ICZ}$ & Poor growth & $\begin{array}{l}\text { Aspergillus niger contamination; but } \\
\text { other fungi is inhibited }\end{array}$ & $2 \times 10^{-5}$ \\
\hline $\begin{array}{l}\text { Air dried at } \\
\mathrm{RT}+\text { heated in } \\
\text { water bath for } \\
60{ }^{\circ} \mathrm{C}\end{array}$ & $\mathrm{AIA}+\mathrm{CHL}+\mathrm{ICZ}$ & Poor growth & $\begin{array}{l}\text { Aspergillus niger contamination; } \\
\text { but other fungi is inhibited }\end{array}$ & $2 \times 10^{-5}$ \\
\hline $\begin{array}{l}\text { Sundried }+ \\
\text { heated in water } \\
\text { bath for } 60^{\circ} \mathrm{C}\end{array}$ & $\mathrm{SCA}+\mathrm{CHL}+\mathrm{ICZ}$ & Good & $\begin{array}{l}\text { Aspergillus and fungal contamination } \\
\text { is restricted to corner of plate }\end{array}$ & $6 \times 10^{-5}$ \\
\hline $\begin{array}{l}\text { Sundried+ } \\
\text { heated in water } \\
\text { bath for } 60^{\circ} \mathrm{C}\end{array}$ & $\mathrm{AIA}+\mathrm{CHL}+\mathrm{ICZ}$ & Good & $\begin{array}{l}\text { Aspergillus and fungal contamination } \\
\text { is restricted to corner of plate }\end{array}$ & $5 \times 10^{-5}$ \\
\hline
\end{tabular}


A Consolidated Method for Selective Isolation of Actinomycetes Based on Choice of Substrate

\begin{tabular}{|l|l|l|l|l|}
\hline $\begin{array}{l}\text { Chemical } \\
\text { enrichment }\end{array}$ & & & & \\
\hline $\begin{array}{l}\text { Sample } \\
\text { enriched with } \\
\mathrm{CaCO}_{3} \text { for 7 } \\
\text { days and air } \\
\text { dried }\end{array}$ & SCA + CHL+ICZ & Better & Less contamination of other microbes & $7 \times 10^{-5}$ \\
\hline $\begin{array}{l}\text { Sample } \\
\text { enriched with } \\
\begin{array}{l}\text { CaCO } \\
\text { days and air } \\
\text { dried }\end{array}\end{array}$ & AIA+ CHL+ICZ & Better & Less contamination of other microbes & $6 \times 10^{-5}$ \\
\hline
\end{tabular}

Note: $\mathrm{RT}=$ Room Temperature, $\mathrm{SCA}=$ Starch Casein Agar, AIA = Actinomycete Isolation Agar, CHL = Chloramphenicol, $\mathrm{ICZ}=$ Itraconazole.

Table 3: Selective isolation of actinomycetes from cow dung compost

\begin{tabular}{|c|c|c|c|c|}
\hline $\begin{array}{l}\text { Pretreatment } \\
\text { of sample }\end{array}$ & Selective Media & $\begin{array}{l}\text { Growth of } \\
\text { actinomycetes }\end{array}$ & Growth of other microbes & $\begin{array}{l}\text { CFU/gm } \\
\text { of dried } \\
\text { sample }\end{array}$ \\
\hline No treatment & SCA & No growth & Bacterial and fungal contamination & - \\
\hline No treatment & AIA & No growth & Bacterial and fungal contamination & - \\
\hline No treatment & $\mathrm{SCA}+\mathrm{CHL}+\mathrm{ICZ}$ & $\begin{array}{l}\text { No distinct } \\
\text { actinomycetes } \\
\text { appear }\end{array}$ & No bacteria; fungal contamination & - \\
\hline No treatment & $\mathrm{AIA}+\mathrm{CHL}+\mathrm{ICZ}$ & $\begin{array}{l}\text { No distinct } \\
\text { actinomycetes } \\
\text { appear }\end{array}$ & $\begin{array}{l}\text { No bacteria; } \\
\text { fungal contamination }\end{array}$ & - \\
\hline \multicolumn{5}{|l|}{ Dry treatment } \\
\hline Air dried at RT & SCA & No growth & Bacterial and fungal contamination & - \\
\hline Air dried at RT & AIA & No growth & Bacterial and fungal contamination & - \\
\hline Air dried at RT & $\mathrm{SCA}+\mathrm{CHL}+\mathrm{ICZ}$ & Good & $\begin{array}{l}\text { Fungal contamination of Aspergillus } \\
\text { niger }\end{array}$ & $4 \times 10^{-5}$ \\
\hline Air dried at RT & $\mathrm{AIA}+\mathrm{CHL}+\mathrm{ICZ}$ & Good & $\begin{array}{l}\text { Fungal contamination of Aspergillus } \\
\text { niger and Aspergillus }\end{array}$ & $5 \times 10^{-5}$ \\
\hline Sundried & SCA & No growth & Bacterial and fungal contamination & - \\
\hline Sundried & AIA & No growth & Bacterial and fungal contamination & - \\
\hline Sundried & $\mathrm{SCA}+\mathrm{CHL}+\mathrm{ICZ}$ & Poor growth & $\begin{array}{l}\text { Aspergillus niger contamination } \\
\text { restricted to corner of plates, but } \\
\text { other fungi is inhibited }\end{array}$ & $2 \times 10^{-5}$ \\
\hline Sundried & $\mathrm{AIA}+\mathrm{CHL}+\mathrm{ICZ}$ & Poor growth & $\begin{array}{l}\text { Aspergillus niger contamination; } \\
\text { but other fungi is inhibited }\end{array}$ & $2 \times 10^{-5}$ \\
\hline \multicolumn{5}{|l|}{ Wet treatment } \\
\hline $\begin{array}{l}\text { Air dried at RT } \\
+ \text { heated in } \\
\text { water bath for } \\
60{ }^{\circ} \mathrm{C}\end{array}$ & $\mathrm{SCA}+\mathrm{CHL}+\mathrm{ICZ}$ & Good & $\begin{array}{l}\text { Aspergillus niger contamination; but } \\
\text { other fungi is inhibited }\end{array}$ & $4 \times 10^{-5}$ \\
\hline $\begin{array}{l}\text { Air dried at RT+ } \\
\text { heated in water } \\
\text { bath for } 60^{\circ} \mathrm{C}\end{array}$ & $\mathrm{AIA}+\mathrm{CHL}+\mathrm{ICZ}$ & Good & $\begin{array}{l}\text { Aspergillus niger contamination; } \\
\text { but other fungi is inhibited }\end{array}$ & $4 \times 10^{-5}$ \\
\hline $\begin{array}{l}\text { Sundried }+ \\
\text { heated in water } \\
\text { bath for } 60^{\circ} \mathrm{C}\end{array}$ & $\mathrm{SCA}+\mathrm{CHL}+\mathrm{ICZ}$ & Poor & $\begin{array}{l}\text { Less fungal contamination still } \\
\text { growth is poor }\end{array}$ & $2 \times 10^{-5}$ \\
\hline $\begin{array}{l}\text { Sundried+ } \\
\text { heated in water } \\
\text { bath for } 60^{\circ} \mathrm{C}\end{array}$ & $\mathrm{AIA}+\mathrm{CHL}+\mathrm{ICZ}$ & Poor & $\begin{array}{l}\text { Less fungal contamination still } \\
\text { growth is poor }\end{array}$ & $2 \times 10^{-5}$ \\
\hline
\end{tabular}


Tiwari et al., Int. Ann. Sci.; Vol. 11, Issue 1, pp: 10-21, 2021

\begin{tabular}{|l|l|l|l|l|}
\hline $\begin{array}{l}\text { Chemical } \\
\text { enrichment }\end{array}$ & & & \\
\hline $\begin{array}{l}\text { Sample enriched } \\
\text { with } \mathrm{CaCO}_{3} \text { for } \\
7 \text { days and air } \\
\text { dried }\end{array}$ & SCA + CHL+ICZ & Better & $\begin{array}{l}\text { Less contamination of other } \\
\text { microbes }\end{array}$ & $6 \times 10^{-5}$ \\
\hline $\begin{array}{l}\text { Sample enriched } \\
\text { with } \mathrm{CaCO}_{3} \text { for } \\
7 \text { days and air } \\
\text { dried }\end{array}$ & AIA+ CHL+ICZ & Better & $\begin{array}{l}\text { Less contamination of other } \\
\text { microbes }\end{array}$ & $5 \times 10^{-5}$ \\
\hline
\end{tabular}

Note: RT= Room Temperature, SCA = Starch Casein Agar, AIA = Actinomycete Isolation Agar, CHL = Chloramphenicol, $\mathrm{ICZ}=$ Itraconazole.

Table 4: Selective isolation of actinomycetes from floral compost

\begin{tabular}{|c|c|c|c|c|}
\hline $\begin{array}{l}\text { Pretreatment of } \\
\text { sample }\end{array}$ & Selective Media & $\begin{array}{l}\text { Growth of } \\
\text { actinomycetes }\end{array}$ & $\begin{array}{l}\text { Growth of other } \\
\text { microbes }\end{array}$ & $\begin{array}{l}\text { CFU/gm of } \\
\text { dried sample }\end{array}$ \\
\hline No treatment & SCA & No growth & $\begin{array}{l}\text { Bacterial and fungal } \\
\text { contamination }\end{array}$ & - \\
\hline No treatment & AIA & No growth & $\begin{array}{l}\text { Bacterial and fungal } \\
\text { contamination }\end{array}$ & - \\
\hline No treatment & $\mathrm{SCA}+\mathrm{CHL}+\mathrm{ICZ}$ & $\begin{array}{l}\text { No distinct } \\
\text { actinomycetes appear }\end{array}$ & $\begin{array}{l}\text { No bacteria; fungal } \\
\text { contamination }\end{array}$ & - \\
\hline No treatment & $\mathrm{AIA}+\mathrm{CHL}+\mathrm{ICZ}$ & $\begin{array}{l}\text { No distinct } \\
\text { actinomycetes appear }\end{array}$ & $\begin{array}{l}\text { No bacteria; } \\
\text { fungal contamination }\end{array}$ & - \\
\hline \multicolumn{5}{|l|}{ Dry treatment } \\
\hline Air dried at RT & SCA & No growth & $\begin{array}{l}\text { Bacterial and fungal } \\
\text { contamination }\end{array}$ & - \\
\hline Air dried at RT & AIA & No growth & $\begin{array}{l}\text { Bacterial and fungal } \\
\text { contamination }\end{array}$ & - \\
\hline Air dried at RT & $\mathrm{SCA}+\mathrm{CHL}+\mathrm{ICZ}$ & Poor & Fungal contamination & $1 \times 10^{-5}$ \\
\hline Air dried at RT & $\mathrm{AIA}+\mathrm{CHL}+\mathrm{ICZ}$ & Poor & Fungal contamination & $1 \times 10^{-5}$ \\
\hline Sundried & SCA & Poor & $\begin{array}{l}\text { Bacterial and fungal } \\
\text { contamination }\end{array}$ & - \\
\hline Sundried & AIA & Poor & $\begin{array}{l}\text { Bacterial and fungal } \\
\text { contamination }\end{array}$ & - \\
\hline Sundried & $\mathrm{SCA}+\mathrm{CHL}+\mathrm{ICZ}$ & Poor & $\begin{array}{l}\text { Aspergillus niger } \\
\text { contamination restricted to } \\
\text { corner of plates, but other } \\
\text { fungi is inhibited }\end{array}$ & $2 \times 10^{-5}$ \\
\hline Sundried & $\mathrm{AIA}+\mathrm{CHL}+\mathrm{ICZ}$ & Poor & $\begin{array}{l}\text { Aspergillus niger } \\
\text { contamination; } \\
\text { but other fungi is inhibited }\end{array}$ & $2 \times 10^{-5}$ \\
\hline \multicolumn{5}{|l|}{ Wet treatment } \\
\hline $\begin{array}{l}\text { Air dried at RT }+ \\
\text { heated in water } \\
\text { bath for } 60^{\circ} \mathrm{C}\end{array}$ & $\mathrm{SCA}+\mathrm{CHL}+\mathrm{ICZ}$ & Poor & $\begin{array}{l}\text { Aspergillus niger } \\
\text { contamination; but other } \\
\text { fungi is inhibited }\end{array}$ & $3 \times 10^{-5}$ \\
\hline $\begin{array}{l}\text { Air dried at RT+ } \\
\text { heated in water } \\
\text { bath for } 60^{\circ} \mathrm{C}\end{array}$ & AIA +CHL +ICZ & Poor & $\begin{array}{l}\text { Aspergillus niger } \\
\text { contamination; } \\
\text { but other fungi is inhibited }\end{array}$ & $2 \times 10^{-5}$ \\
\hline $\begin{array}{l}\text { Sundried + heated } \\
\text { in water bath for } \\
60^{\circ} \mathrm{C}\end{array}$ & $\mathrm{SCA}+\mathrm{CHL}+\mathrm{ICZ}$ & Poor & $\begin{array}{l}\text { Less fungal contamination } \\
\text { still growth is poor }\end{array}$ & $2 \times 10^{-5}$ \\
\hline $\begin{array}{l}\text { Sundried+ heated } \\
\text { in water bath for } \\
60^{\circ} \mathrm{C}\end{array}$ & $\mathrm{AIA}+\mathrm{CHL}+\mathrm{ICZ}$ & Poor & $\begin{array}{l}\text { Less fungal contamination } \\
\text { still growth is poor }\end{array}$ & $2 \times 10^{-5}$ \\
\hline
\end{tabular}


A Consolidated Method for Selective Isolation of Actinomycetes Based on Choice of Substrate

\begin{tabular}{|l|l|l|l|l|}
\hline $\begin{array}{l}\text { Chemical } \\
\text { enrichment }\end{array}$ & & & & \\
\hline $\begin{array}{l}\text { Sample enriched } \\
\text { with } \mathrm{CaCO}_{3} \text { for } 7 \\
\text { days and air dried }\end{array}$ & SCA + CHL+ICZ & Better & $\begin{array}{l}\text { Less contamination of } \\
\text { other microbes }\end{array}$ & $8 \times 10^{-5}$ \\
\hline $\begin{array}{l}\text { Sample enriched } \\
\text { with } \mathrm{CaCO}_{3} \text { for } 7 \\
\text { days and air dried }\end{array}$ & AIA+ CHL+ICZ & Better & $\begin{array}{l}\text { Less contamination of } \\
\text { other microbes }\end{array}$ & $5 \times 10^{-5}$ \\
\hline
\end{tabular}

Note: RT= Room Temperature, SCA = Starch Casein Agar, AIA = Actinomycete Isolation Agar, CHL = Chloramphenicol, ICZ = Itraconazole.

Table 5: Selective isolation of actinomycetes from floral vermicompost

\begin{tabular}{|c|c|c|c|c|}
\hline $\begin{array}{l}\text { Pretreatment of } \\
\text { sample }\end{array}$ & Selective Media & $\begin{array}{l}\text { Growth of } \\
\text { actinomycetes }\end{array}$ & Growth of other microbes & $\begin{array}{l}\text { CFU/gm of } \\
\text { dried } \\
\text { sample }\end{array}$ \\
\hline No treatment & SCA & No growth & $\begin{array}{l}\text { Fluoroscence Pseudomonas } \\
\text { sp. and other bacteria, no } \\
\text { fungi }\end{array}$ & - \\
\hline No treatment & AIA & No growth & $\begin{array}{l}\text { Fluoroscence Pseudomonas } \\
\text { sp. and other bacteria, no } \\
\text { fungi }\end{array}$ & - \\
\hline No treatment & $\mathrm{SCA}+\mathrm{CHL}+\mathrm{ICZ}$ & $\begin{array}{l}\text { No distinct } \\
\text { actinomycetes appear }\end{array}$ & $\begin{array}{l}\text { Fluoroscence Pseudomonas } \\
\text { sp., no other bacteria and } \\
\text { fungi }\end{array}$ & - \\
\hline No treatment & $\mathrm{AIA}+\mathrm{CHL}+\mathrm{ICZ}$ & $\begin{array}{l}\text { No distinct } \\
\text { actinomycetes appear }\end{array}$ & $\begin{array}{l}\text { Fluoroscence Pseudomonas } \\
\text { sp., no other bacteria and } \\
\text { fungi }\end{array}$ & - \\
\hline \multicolumn{5}{|l|}{ Dry treatment } \\
\hline Air dried at RT & SCA & No growth & $\begin{array}{l}\text { Fluroscence Pseudomonas } \\
\text { sp.growth inhibiting fungi. } \\
\text { No other bacterial } \\
\text { contamination }\end{array}$ & - \\
\hline Air dried at RT & AIA & No growth & $\begin{array}{l}\text { Fluroscence Pseudomonas } \\
\text { sp.growth inhibiting fungi. } \\
\text { No other bacterial } \\
\text { contamination }\end{array}$ & - \\
\hline Air dried at RT & $\mathrm{SCA}+\mathrm{CHL}+\mathrm{ICZ}$ & No growth & $\begin{array}{l}\text { Fluroscence Pseudomonas } \\
\text { sp. growth inhibiting } \\
\text { Aspergillus spp. No other } \\
\text { bacteria and fungi }\end{array}$ & - \\
\hline Air dried at RT & $\mathrm{AIA}+\mathrm{CHL}+\mathrm{ICZ}$ & No growth & $\begin{array}{l}\text { Fluroscence Pseudomonas } \\
\text { sp.growth inhibiting } \\
\text { Aspergillus spp. No other } \\
\text { bacteria and fungi }\end{array}$ & - \\
\hline Sundried & SCA & No growth & $\begin{array}{l}\text { Fluroscence Pseudomonas } \\
\text { sp. growth inhibiting fungi. } \\
\text { No other bacterial } \\
\text { contamination }\end{array}$ & - \\
\hline Sundried & AIA & No growth & $\begin{array}{l}\text { Fluroscence Pseudomonas } \\
\text { sp. growth inhibiting fungi. } \\
\text { No other bacterial } \\
\text { contamination }\end{array}$ & - \\
\hline Sundried & $\mathrm{SCA}+\mathrm{CHL}+\mathrm{ICZ}$ & No growth & $\begin{array}{l}\text { Fluroscence Pseudomonas } \\
\text { sp. }\end{array}$ & - \\
\hline Sundried & $\mathrm{AIA}+\mathrm{CHL}+\mathrm{ICZ}$ & No growth & $\begin{array}{l}\text { Fluroscence Pseudomonas } \\
\text { sp. }\end{array}$ & - \\
\hline
\end{tabular}


Tiwari et al., Int. Ann. Sci.; Vol. 11, Issue 1, pp: 10-21, 2021

\begin{tabular}{|c|c|c|c|c|}
\hline Wet treatment & & & & \\
\hline $\begin{array}{l}\text { Air dried at RT }+ \\
\text { heated in water } \\
\text { bath for } 60^{\circ} \mathrm{C}\end{array}$ & $\mathrm{SCA}+\mathrm{CHL}+\mathrm{ICZ}$ & No growth & $\begin{array}{l}\text { Fluroscence Pseudomonas } \\
\text { sp. }\end{array}$ & - \\
\hline $\begin{array}{l}\text { Air dried at RT+ } \\
\text { heated in water } \\
\text { bath for } 60^{\circ} \mathrm{C}\end{array}$ & $\mathrm{AIA}+\mathrm{CHL}+\mathrm{ICZ}$ & No growth & $\begin{array}{l}\text { Fluroscence Pseudomonas } \\
\text { sp. }\end{array}$ & - \\
\hline $\begin{array}{l}\text { Sundried }+ \text { heated } \\
\text { in water bath for } \\
60^{\circ} \mathrm{C}\end{array}$ & $\mathrm{SCA}+\mathrm{CHL}+\mathrm{ICZ}$ & No growth & $\begin{array}{l}\text { Fluroscence Pseudomonas } \\
\text { sp. }\end{array}$ & - \\
\hline $\begin{array}{l}\text { Sundried+ heated } \\
\text { in water bath for } \\
60^{\circ} \mathrm{C}\end{array}$ & $\mathrm{AIA}+\mathrm{CHL}+\mathrm{ICZ}$ & No growth & $\begin{array}{l}\text { Fluroscence Pseudomonas } \\
\text { sp. }\end{array}$ & - \\
\hline $\begin{array}{l}\text { Chemical } \\
\text { enrichment }\end{array}$ & & & & \\
\hline $\begin{array}{l}\text { Sample enriched } \\
\text { with } \mathrm{CaCO}_{3} \text { for } 7 \\
\text { days and air dried }\end{array}$ & $\mathrm{SCA}+\mathrm{CHL}+\mathrm{ICZ}$ & Better & $\begin{array}{l}\text { Less contamination of other } \\
\text { microbes }\end{array}$ & $8 \times 10^{-5}$ \\
\hline $\begin{array}{l}\text { Sample enriched } \\
\text { with } \mathrm{CaCO}_{3} \text { for } 7 \\
\text { days and air dried }\end{array}$ & $\mathrm{AIA}+\mathrm{CHL}+\mathrm{ICZ}$ & Better & $\begin{array}{l}\text { Less contamination of other } \\
\text { microbes }\end{array}$ & $7 \times 10^{-5}$ \\
\hline
\end{tabular}

Note: RT= Room Temperature, $\mathrm{SCA}=$ Starch Casein Agar, $\mathrm{AIA}=$ Actinomycete Isolation Agar, $\mathrm{CHL}=$ Chloramphenicol, $\mathrm{ICZ}=$ Itraconazole.

In case of vermicompost of floral waste however, growth of actinomycetes was only achieved with enrichment of sample with $\mathrm{CaCO}_{3}$ (Table 4). Colony color of actinomycetes strains isolated was categorized into puffy white, grayish brown, brownish, brownish black, grey centered white (Fig. 8). Actinomycetes colonies were identified through microscopical techniques showing filamentous hyphae and spores morphology. All the isolates were showing spore chain morphology and were identified as Streptomyces sp (Fig.9). In case of floral vermicompost, the colonies of actinomycetes appeared only after $\mathrm{CaCO}_{3}$ enrichment (Table 5). In other pretreatment methods there was dominance of Pseudomonas sp. with fluorescence appearance (Fig 6). The presence of Psendomonas was confirmed when purified on nutrient agar and SCA. Both the plates turned green within $24 \mathrm{hrs}$ incubation and oxidase test was found to be positive as per Bergey's Manual of Determinative Bacteriology (Holt et al., 1994). It was found that these colonies have secreted some volatile substances when plates were under incubation for 7-8 days and have killed the Aspergillus sp. and other fungi like colonies (Fig 7). A kind of pungent smell was detected on these agar plates. Only in case of soil sample $10^{-6}$ dilution was preferable to get countable colonies. Rest in other cases dilution up to $10^{-3}$ gave expected results.

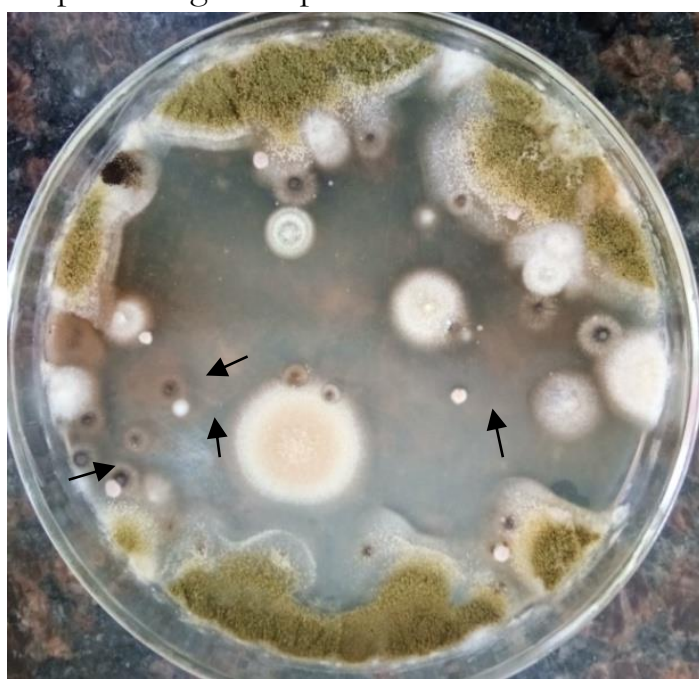

Figure 3: Pretreated sample (Physical) actinomycetes grown on plate

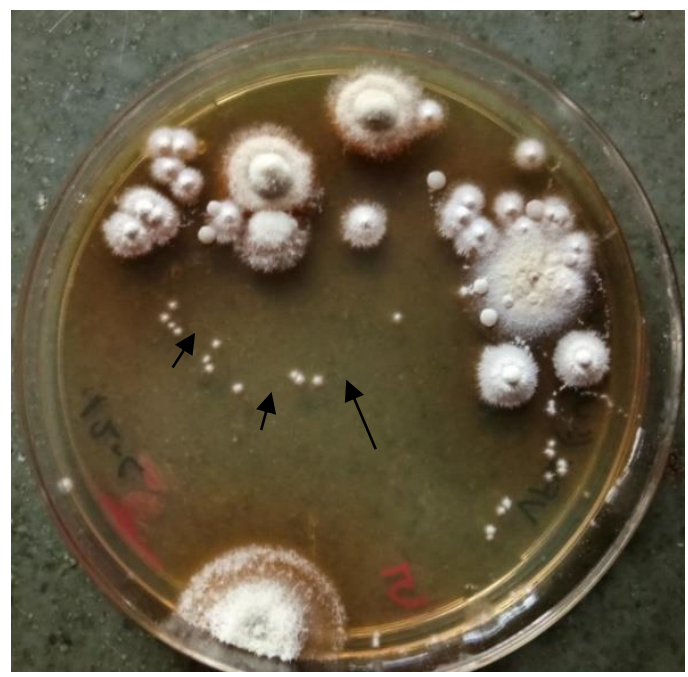

Figure 4: Pretreated sample ( $\mathrm{CaCO}_{3}$ enrichment) 


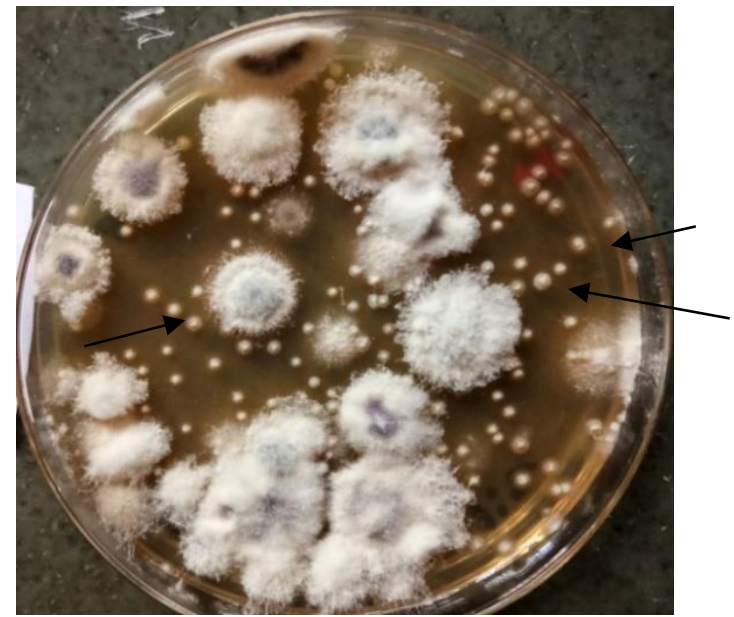

Figure 5: Pretreated sample $\left(\mathrm{CaCO}_{3}\right.$ enrichment showing better growth of actinomycetes and more in number

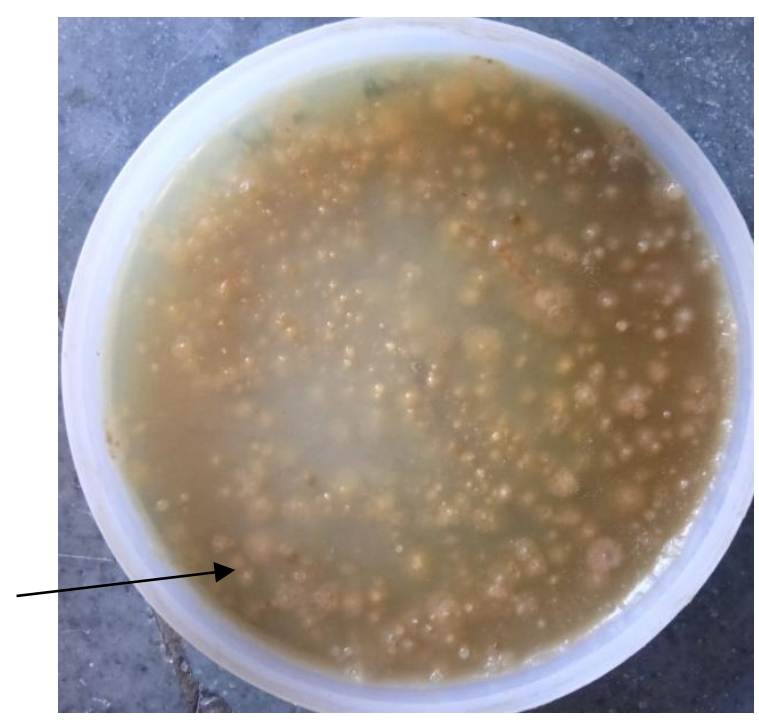

Figure 6: Greenish colony of fluorescent Pseudomonas sp. covers the whole plate.

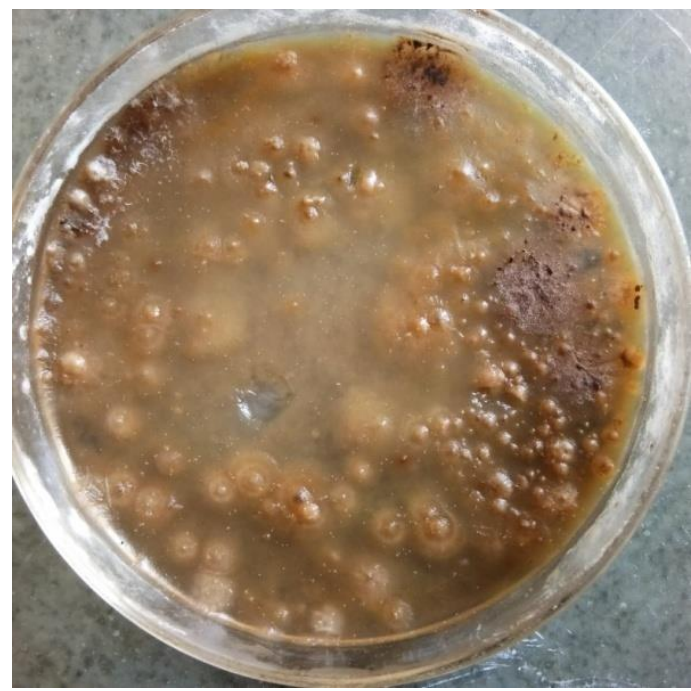

Figure 7: Fluorescent Pseudomonas killed fungus after 7 days 
Tiwari et al., Int. Ann. Sci.; Vol. 11, Issue 1, pp: 10-21, 2021

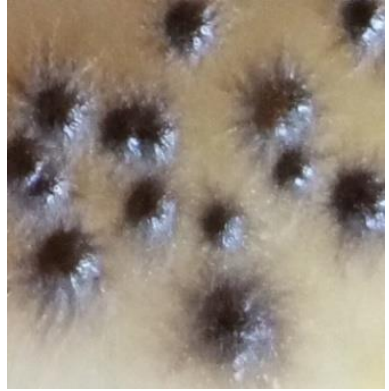

(a)

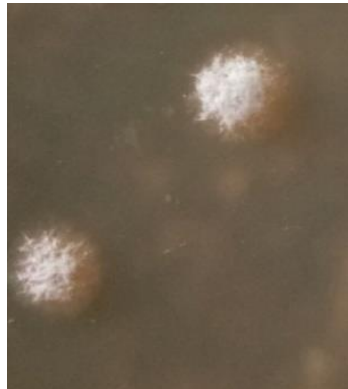

(b)

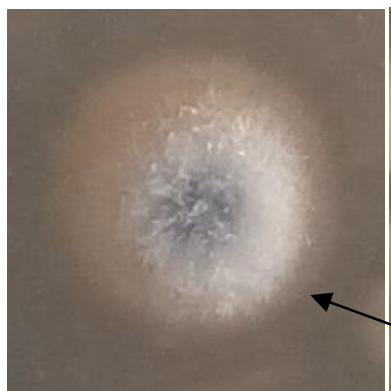

(c)

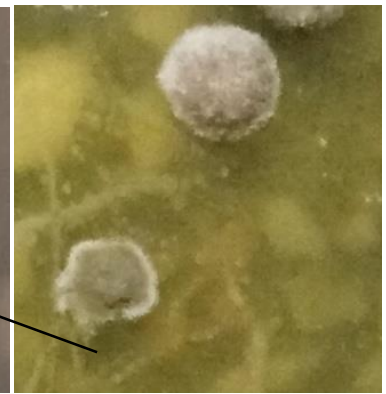

(d)

Figure 8: Color of some of the purified colonies a) Blackish brown b) white puffy c) grayish centered white d) chalky brownish

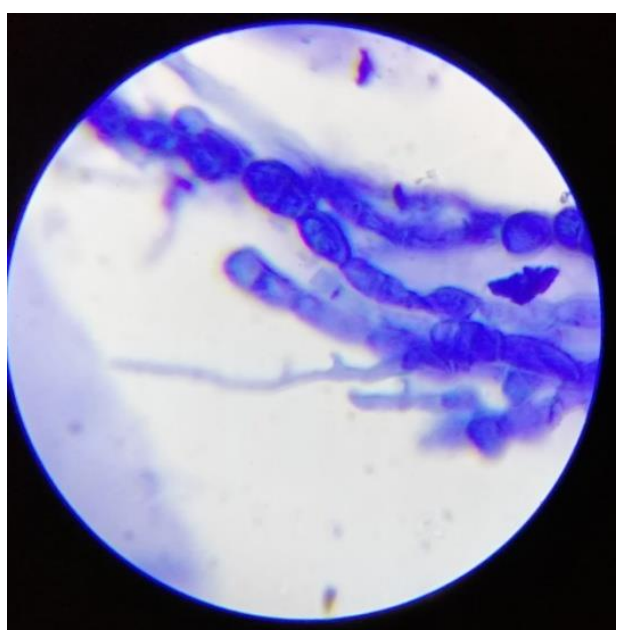

Figure 9: Microscopic view of some of the actinomycetes isolates showing spore chain morphology

In the entire above cases SCA agar and AIA agar gave almost same results. However, Aspergillus sp. contamination was more in AIA agar then SCA. Total 12 isolates were obtained with different colour colony. All the 12 presumed actinomycetes isolated were coded as ACT 1-12 and tested under microscope under 10X and 100X showed filaments and spore chains characteristics.

\section{Discussions}

From the above results it is clear that pretreatment of sample and growth of actinomycetes depends on choice of substrate or source from which sample was collected (Khama et al., 2011; Chavan et al., 2013). In case of soil since it constitutes mixed flora in large quantity both wet and moist treatment required to achieve isolation of actinomycetes. In case of cow dung compost a different type of black colored colonies was observed of filamentous nature. It is better achieved in air dried sample than in sun dried one. This may be due to sun drying the mesophillic actinomycetes growth has been restricted since compost was maintained in moist condition and room temperature around 30-32 C. So, this may have provided good favorable niche for mesophillic actinomycetes to grow. In case of floral waste, the growth was achieved well only when enriched with $\mathrm{CaCO}$. This may be due to rare actinomycetes genera inhabiting the niche in the maturing phase of composting and thus need to be enriched for their isolation from mixed population of microbes (Hayakawa, 2008). Vermicompost of floral waste on the other hand showed the presence of a type of Pseudomonas sp. Colonies appears fluorescent and transparent same as color of media in 24 hours and turns to green in next $24 \mathrm{hrs}$ with pungent smell. The similar results were observed from vermicompost reported by Sequeria and Chandrashekhar, 2015. Involvement of HCN produced by fluorescent Pseudomonas in disease suppression by induction of plant resistance has been reported by Voisard et al., 1981 . HCN production by Pseudomonas fluorescens is reported to suppress black root rot of tobacco (Hass et al., 1991). The suppression of Aspergillus and other fungi in our case by fluorescent Pseudomonas sp. may be 
due production of HCN. However, the presence of such species only in vermicompost samples may be due to the presence of such species in digestive tract of earthworms (Vega and Victoria, 2009) which later have come out of earthworms along with the castings and might have multiplied rapidly as compared to slow growing actinomycetes and thus dominated the other flora and same results were appeared on agar medium too. On chemical enrichment the production of volatile substance by Pseudomonas sp. might have inhibited due to change in $\mathrm{pH}$ and thus it might have favored the isolation of actinomycetes. Talking about dilution factor only in case of soil sample $10^{-6}$ dilution was preferable to get countable colonies. Rest in other cases dilution up to $10^{-3}$ gave expected results. This may be due abundance of micro flora in soil in vegetative or spore forms constituting gram negative, gram positive bacteria, fungi, actinomycetes hence to selectively isolate actinomycetes from soil $10^{-6}$ dilution is preferable to get countable colonies else it will give massive growth on less dilution factor. However, in case of composts the dominance of actinomycetes mainly occurs at later stages since there is succession of microbial colonies; bacteria, fungi, actinomycetes (Rebollido et al., 2008), hence dilution factor up to $10^{-3}$ can give expected results. Higher dilution factor in this case did not give good results. During the whole experiment except in case of vermicompost samples which shows fluorescent Psendomonas colonies rest all samples though on pretreatment and enrichment showed actinomycetes colonies on media supplemented with antibiotics still they were not able to inhibit the growth of fungi completely. There is mainly contamination of Aspergillus sp. This may be due to resistance of some of the fungal species to these antifungal drugs. Growth of non-actinomycetes bacteria was however completely inhibited. Hence use of antibiotics can reduce the number of fungi but cannot completely suppress them.

\section{Conclusion}

From the present study we can conclude that selective isolation of actinomycetes can be done effectively by combining pretreatment either physical or chemical or both along with suitable media supplemented with specific antibiotics. The range of pretreatment methods can be applied depending on the substrate chosen in study. Though $80 \%$ of the actinomycetes comprise Streptomyces and can be easily isolated and pretreatment is mainly required to isolate rare genera of actinomycetes as mentioned by many researchers. But from our study we conclude that a consolidated approach is needed for better results. One should remember that our study was quantitative in nature therefore, there is a possibility of different ways of pretreatment methods might have favored the repeated isolation of same types of actinomycetes. This can be judged simply by morphological characteristics and microscopy. The isolates with distinct morphology after purification and microscopic characteristics can be selected for further studies. The study also demonstrated that not only soils but other niches such as manure composts, floral waste composts, vermicomposts etc. can be utilized for isolation of actinomycetes as an addition source for the screening of novel bioactive compounds.

\section{Declarations}

\subsection{Acknowledgement}

Authors are very thankful to our Principal, Govt. Madhav Science, P.G. College, Ujjain, (M.P) for giving his help and support.

\subsection{Competing Interests}

The authors have no conflicts of interest to declare.

\section{How to Cite this Article:}

D. Tiwari, S. Shouche, P. Bhati, and P. Das, "A Consolidated Method for Selective Isolation of Actinomycetes Based on Choice of Substrate”, Int. Ann. Sci., vol. 11, no. 1, pp. 10-21, Dec. 2021. 
Tiwari et al., Int. Ann. Sci.; Vol. 11, Issue 1, pp: 10-21, 2021

\section{References}

[1] Anusuya,. D and Geetha, M. Isolation of Thermophilic Actinomycetes from Banana Waste Compost and their Biochemical Characteristics.International Journal of Science and Research. 2014; 3(5): 315-317

[2] Arifuzzaman, M., Khatun, M.R., Rahman, H. Isolation and Screening of Actinomycetes from Sundarbans Soil for Antibacterial Activity. African Journal of Biotechnology. 2010; 9(29): 4615-4619.

[3] Ashraf, R., Shahid, F., Ali, T.A. Association of Fungi, Bacteria and Actinomycetes with Different Composts. Pak.J.Bot. 2007; 39(6): 2141-2151

[4] Attimarad, S.L., Ediga, G.N., Karigar, A.A., Karadi, R., Chandrashekhar, N. Screening and Isolation and Purification of Antibacterial Agents from Marine Actinomycetes. International Current Pharmaceutical Journal. 2012; 1(2): 394-402.

[5] Bhasin, S. and Modi, H.A. Streptomycetes: A Storehouse of Bioactive Compounds and Enzymes. A Production of Glucose Isomerase. Research Journal of Recent Sciences. 2013; 2: 330-339.

[6] Chaudhary, H.S, Yadav, J., Shrivastav, A.R., Singh, A.K., Gopalan, N. Antibacterial activity of Actinomycetes Isolated from Different Soil Samples of Sheopur (A city of Central India). Journal of Advanced Pharmaceutical Technology and Research. 2013; 4(2): 118123.

[7] Chavan D.V., Mulaje, S.S., Mohalkar R.Y. A Review on Actinomycetes and Their Biotechnological Applications. International Journal of Pharmaceutical Sciences and Research. 2013; 4(5): 1730 - 1742.

[8] El-Nakeeb,M.A., and Lechevalier. H.A. Selective Isolation of Aerobic Actinomycetes. Applied Microbiology.1962:75-77.

[9] Gopalakrishnan, S., Pande., S., Sharma, M., Humayun, P., Kiran, B.K., Sandeep, D., Vidya, M.S., Deepthi, K., Rupela, O. Evaluation of Actinomycete Isolates obtained from Herbal Vermicompost for the Biological Control of Fusarium wilt of Chickpea. International Crops Research Institute for Semi-Arid Tropics. 2011: 01-29.

[10] Hayakawa, M., Yamamura, H., Nakagawa, Y., Kawa, Y., Hayashi, Y., Misonou, T., Kaneko, H., Kikushima, N., Takahashi, T., Yamasaki, S., Uegaki, Y., Terasawa, A., Takao, K., Mori, T., Ago, K., Saito, N. and Hasegawa, T. Taxonomic Diversity of Actinomycetes Isolated from Swine Manure Compost. Actinomycetologica. 2010; 24 (2): 58-62.

[11] Iyer, K., and Subramanyam, S.K. Isolates were characterized by using morphological and biochemical methods. International Journal of Science, Technology \& Management.2014; 3(3): 25-36

[12] Jeffrey, L. S. H. Isolation, characterization and identification of actinomycetes from agriculture soils at Semongok, Sarawak. African Journal of Biotechnology. 2008; 7(20): 3697-3702.

[13] Kumar, R.R. and Jadeja, V.J. Isolation of Actinomycetes: A Complete Approach. International Journal of Current Microbiology and Applied Sciences. 2016; 5(5): 608-618.

[14] Kumar, V., Bharti, A., Negi, Y.K., Gusain, O., Pandey, P., Bisht, G.S. Screening of Actinomycetes from Earthworm castings for their antimicrobial activity and industrial enzymes. Brazilian Journal of Microbiology.2012; 43(1): 01-07.

[15] Lekshmi, M., Jayadev, A. and Navami, S.S. Isolation and Screening of Actinomycetes from Marine Samples for Enzyme Production. International Journal of Scientific and Engineering Research. 2014; 5(12): 199-204.

[16] Limaye, L., Patil, R., Ranadive, P., Kamath, G.Application of Potent Actinomycete Strains for Bio-Degradation of Domestic AgroWaste by Composting and Treatment of Pulp-Paper Mill Effluent. Advances in Microbiology. 2017; 7: 94-108

[17] Lo, C.W., Lai, N.S., Cheah, H.Y., Wong, N.K.I., Ho, C.C. Actinomycetes isolated from soil samples from the Crocker Range Sabah. ASEAN Review of Biodiversity and Environmental Conservation. 2002; 1-7.

[18] Malisorn, K. and Nikhome, K. Isolation and screening of Actinomycetes from soil for their enzymatic and antifungal activity. Khon Kaen Agr. J. 42 Suppl. 4. 2014, 151-156.

[19] Peela, S., and Porana, S. Isolation and screening of novel streptomyces from sediments of Bay of Bengal near Srikulam coast. International Journal of Current Pharmaceutical Research. 2017; 9(1): 40-44.

[20] Rebollido, R., Martinez, J., Aguilera,Y., Melchor, K., Koerner, I., Stegmann, R. Microbial populations during composting process of organic fraction of municipal solid waste. Applied Ecology and Environmental Research. 2008; 6(3): 61-67.

[21] Shirling, E.B. and Gottlieb, D. Methods for characterization of Streptomyces species. International Journal of Systematic Bacteriology. 1966; 16(3): 313-340.

[22] Tsao, P.H., Leben,C., and Keitt, G.W. An enrichment method for isolating actinomycetes that produce diffusible antifungal antibiotics. Phytopathology. 1960; 50:88-89.

[23] Vega, H.B., and Victoria, D.E. Bacterial diversity in the digestive tract of Earthworms (Oligochaeta). Journal of Biological Sciences. 2009; 9(3):192-193

[24] Voisard, C., Keel, C., Haas, D., Defago. G. Cyanide production by Pseudomonas flurorescens helps suppress black root rot of tobacco under gnotobiotic conditions. EMBO Journal.1989; 8:351.

[25] Williams, S.T., and Cross, T. Actinomycetes isolation from soil, Methods in microbiology, Academic press, London, New York. 1971; 4: 295-334.

[26] Williams, S.T., Goodfellow, M., Alderson, G., Wellington, E.M.H., Sneath, P.H. and Sakin, M.J. Numerical classification of Streptomyces and related genera. J. Gen. Microbiol. 1983; 129: 1743 - 1813.

[27] Workie, M. and Abate, D. Screening of Bioactive Compounds from Actinomycetes Isolated from Compost Prepared for Mushroom Growth against Candida albicans. International Journal of Innovative Pharmaceutical Sciences and Research. 2016; 4(8), 887-889.

[28] Yami, K.D., Bhattarai, S. and Adhikari, S. Vermicomposting and Micro flora Analysis of Vermicompost, Vermicast and Gut or Red Earthworm. Nepal Journal of Science and Technology. 2003; 5:121-126.

Publish your research article in AIJR journals-

$\checkmark$ Online Submission and Tracking

$\checkmark$ Peer-Reviewed

$\checkmark$ Rapid decision

$\checkmark$ Immediate Publication after acceptance

$\checkmark$ Articles freely available online

$\checkmark \quad$ Retain full copyright of your article.

Submit your article at journals.aijr.org
Publish your books with AIJR publisherPublish with ISBN and DOI. Publish Thesis/Dissertation as Monograph. Publish Book Monograph. Publish Edited Volume/ Book. Publish Conference Proceedings Retain full copyright of your books. Submit your manuscript at books.aijr.org 